\title{
Positron emission tomography combined with diagnostic chest computed tomography enhances detection of regional recurrence after stereotactic body radiation therapy for early stage non-small cell lung cancer
}

\author{
Michael I. Ebright, MD, ${ }^{a}$ Gregory A. Russo, MD, ${ }^{\mathrm{b}}$ Avneesh Gupta, MD, ${ }^{\mathrm{c}}$ Rathan M. Subramaniam, MD, ${ }^{\mathrm{b}, \mathrm{c}}$ \\ Hiran C. Fernando, MBBS, ${ }^{a}$ and Lisa A. Kachnic, MD $^{\mathrm{b}}$
}

\begin{abstract}
Objective(s): Recommendations for surveillance after stereotactic body radiation therapy (SBRT) for early stage non-small cell lung cancer (NSCLC) are not well defined. Prospective studies evaluating the efficacy of SBRT have used interval posttreatment imaging with computed tomography (CT). We set out to determine whether positron emission tomography (PET) combined with diagnostic chest CT (PET/d-chest) can enhance detection of potentially salvageable recurrence after SBRT.
\end{abstract}

\begin{abstract}
Methods: We performed a retrospective analysis of posttreatment imaging for 35 patients consecutively treated with SBRT for biopsy-proven early-stage NSCLC. PET/d-chest was generally performed every 3 months after treatment. A board-certified radiologist who did not have access to the PET results retrospectively interpreted the CT scans. CT results were reported according to response criteria used in Radiation Therapy Oncology Group 0236 and compared with PET/d-chest readings. Local and regional recurrence-free survival was compared using the Mantle-Cox (log-rank) test.
\end{abstract}

Results: Median follow-up was 12.8 months. Twenty-four patients had stage IA, 7 stage IB, 3 stage IIA, and 1 stage IIB biopsy-proven NSCLC. Two-year overall survival was $62 \%$. CT scans indicated no regional recurrences. PET/d-chest indicated 10 regional recurrences. The 1-year rate of regional recurrence-free survival as evaluated by CT and PET/d-chest was $100 \%$ and $69.4 \%$, respectively $(P=.0045)$. Four of 10 patients with a diagnosis of regional recurrence underwent salvage treatment with definitive chemoradiotherapy.

Conclusions: PET/d-chest enhances the detection of regional progression of NSCLC after SBRT over currently recommended practices. In patients who are fit for salvage treatment, where early detection of recurrence can increase the likelihood of successful treatment, PET/d-chest appears critical for follow-up. (J Thorac Cardiovasc Surg 2013;145:709-15)

\section{Earn CME credits at}

http://cme.ctsnetjournals.org

Stereotactic body radiation therapy (SBRT) is emerging as the preferred treatment modality for patients with medically inoperable peripheral stage I non-small cell lung cancer (NSCLC). The safety and efficacy of SBRT

\footnotetext{
From the Departments of Thoracic Surgery, ${ }^{\mathrm{a}}$ Radiation Oncology, ${ }^{\mathrm{b}}$ and Radiology, Boston Medical Center and Boston University School of Medicine, Boston, Mass. This work was supported in part by the DOD 06131010 grant (B.U.) (P.I. Kachnic). Disclosures: Authors have nothing to disclose with regard to commercial support. Read at the 38th Annual Meeting of The Western Thoracic Surgical Association, Maui, Hawaii, June 27-30, 2012.

M.I.E. and G.A.R. contributed equally to this research effort.

Received for publication June 18, 2012; revisions received Oct 24, 2012; accepted for publication Dec 10, 2012; available ahead of print Jan 14, 2013.

Address for reprints: Michael I. Ebright, MD, Cardiothoracic Surgery, Boston University School of Medicine, 88 East Newton St, B-402, Boston, MA 02118 (E-mail: michael.ebright@bmc.org).

$0022-5223 / \$ 36.00$

Copyright (c) 2013 by The American Association for Thoracic Surgery

http://dx.doi.org/10.1016/j.jtcvs.2012.12.024
}

have been systematically evaluated in phase I and II clinical trials ${ }^{1-4}$ and shown to have excellent response rates, with local control and survival superior to historically compared results for conventionally fractionated radiation therapy. ${ }^{5}$

Inasmuch as new multicenter clinical trials may expand the indications of SBRT to high-risk patients with operable disease, questions remain regarding the optimal surveillance protocol after treatment. Neither National Comprehensive Cancer Network (NCCN) nor the American Society for Therapeutic Radiology and Oncology (ASTRO) clinical practice guidelines for SBRT of lung cancer make specific recommendations for follow-up after SBRT. ${ }^{6}$ Phase II trials have typically included 3-month interval posttreatment imaging: alternating computed tomography (CT) and chest radiography. ${ }^{7}$ Recent Radiation Therapy Oncology Group (RTOG) studies of SBRT have not routinely included the use of positron emission tomography (PET) for followup owing to concerns that flurodeoxyglucose (FDG) activity persists after treatment for up to 2 years, limiting its utility to assess in-field recurrence. ${ }^{89}$ However, because many failures occur within regional lymphatic basins (ie, hilar 


\section{Abbreviations and Acronyms \\ ACOSOG = American College of Surgeons Oncology Group \\ ASTRO = American Society for Therapeutic Radiology and Oncology
CT $=$ computed tomography
EBUS = endobronchial ultrasound
FDG = fluorodeoxyglucose
NCCN $=$ National Comprehensive Cancer Network \\ NSCLC = non-small cell lung cancer \\ PET = positron emission tomography \\ $\mathrm{PET} / \mathrm{d}$-chest $=$ positron emission tomography combined with diagnostic chest computed tomography
RECIST $=$ Response Evaluation Criteria in Solid Tumors \\ rRFS = regional recurrence-free survival \\ RTOG = Radiation Therapy Oncology Group \\ SBRT = stereotactic body radiation therapy}

and mediastinal), it is critical that these recurrences be detected as early as possible in patients who may be able to tolerate additional salvage treatment. Concern exists that basic anatomic imaging is inadequate in this population, and a prior prospective study has shown that PET/CT performed 3 months after conventionally fractionated definitive radiation therapy discovered potentially salvageable disease in asymptomatic patients more frequently than $\mathrm{CT}$ alone. ${ }^{10}$

In recognition of the relative insensitivity of CT for detecting mediastinal nodal recurrences because of its reliance on morphology and size, ${ }^{11}$ and the superiority of combined PET/CT, which uses metabolic and morphologic information, ${ }^{12}$ we opted to monitor treated patients with diagnostic level chest CT using intravenous contrast in combination with 18-FDG-PET imaging (PET/d-chest) in 3-month intervals. We observed that most (if not all) of the regional (ie, hilar/mediastinal nodal) recurrences detected were done so by the PET portion of the PET/d-chest study. We therefore hypothesized that the addition of PET imaging to diagnostic chest $\mathrm{CT}$ increased the sensitivity of regional recurrence detection and enhanced early discovery of salvageable disease. As such, we performed a retrospective review of the patients treated at our institution to determine the relative utility of diagnostic chest CT and PET/d-chest for surveillance of patients treated with SBRT for early-stage NSCLC.

\section{PATIENTS AND METHODS \\ Patients}

After obtaining institutional review board approval, including the waiver of individual patient consent, we conducted a retrospective review of 40 patients with medically inoperable disease consecutively treated with SBRT for biopsy-proven early stage (T1a-T3, N0 M0) NSCLC at Boston Medical Center from February 2009 until February 2012. Follow-up imaging was only available for 35 of the 40 patients. Of the 5 patients not analyzed, 3 refused to travel to our institution for follow-up and were seen by their referring oncologists close to their homes, 1 died 4 weeks after treatment of a myocardial infarction, and 1 had brain metastases develop before the first scheduled follow-up imaging. The baseline characteristics for these 35 patients are presented in Table 1 . Medical inoperability was determined by board-certified thoracic surgeons (M.I.E., H.C.F.). It should be noted that forced expiratory volume in 1 second and/or diffusing capacity of carbon monoxide of less than $40 \%$ predicted was common, but certainly not sufficient, to conclude medical inoperability. Included were 6 surgical refusals.

Pretreatment assessment and staging were accomplished with diagnostic chest CT with intravenous contrast, PET/CT, imaging of the central nervous system with either contrast-enhanced brain magnetic resonance imaging or head CT, and in select cases, invasive mediastinal/hilar staging with cervical mediastinoscopy and/or endobronchial ultrasound (EBUS) guided lymph node biopsies. After evaluation, all patients were believed to have no regional lymph node metastases. In all patients, staging was according to the seventh edition of the American Joint Commission on Cancer Staging Manual. Tissue confirmation of NSCLC was obtained in all patients and gold fiducial markers were typically placed at the time of biopsy.

\section{SBRT Delivery}

All SBRT treatments were delivered at our institution on a CyberKnife Robotic Stereotactic Radiosurgery System (Accuray, Inc, Sunnyvale, Calif). Patients were positioned for treatment in a body-length vacuum cushion with arms down for comfort. Treatment planning was done with noncontrast chest CT performed in the radiology department on a GE Lightspeed 64 Slice CT scanner (GE Medical Imaging, Waukeshka, Wis). Images were imported into the MultiPlan CyberKnife treatment planning system (Accuray, Inc, Sunnyvale, Calif).

The gross tumor volume was defined by the treating radiation oncologist and thoracic surgeon as the tumor visible on lung windowed CT scan. A margin of at least $5 \mathrm{~mm}$ was added to the gross tumor volume to define the planning target volume. Relevant critical structures (eg, lungs, spinal cord, proximal bronchial tree, esophagus) were also defined. Treatment plans were formulated to treat at least $95 \%$ of the planning target volume with 50 to $60 \mathrm{~Gy}$ in 3 to 5 fractions with the biologically equivalent dose greater than $100 \mathrm{~Gy}_{10}$ for every treatment course. The first 24 treatment plans were calculated with the Ray-Tracing algorithm, and the subsequent 11 were calculated with the Monte Carlo algorithm. Prescription isodose line was between $60 \%$ and $90 \%$. Radiation dose to critical structures was limited to acceptable and safe levels.

All treatments were delivered with image guidance using implanted fiducial markers as surrogates for tumor location, and the Synchrony Respiratory Tracking System (Accuray, Inc, Sunnyvale, Calif) was used for all cases. Patients were pretreated with $4 \mathrm{mg}$ of dexamethasone on days of treatment and SBRT treated on nonconsecutive days.

\section{Radiographic Assessment and Follow-up}

Follow-up plan on completion of SBRT included every 3-month PET/dchest for the first 2 years. In the event that a recurrence was detected on $\mathrm{PET} / \mathrm{d}$-chest, pathologic confirmation was pursued unless a patient was believed too frail to tolerate any salvage or palliative treatment. Pathologic confirmation of mediastinal and/or hilar lymph node recurrence was performed with cervical mediastinoscopy, EBUS, and/or endoscopic esophageal ultrasound.

All PET/CT studies were performed on a GE Discovery STE 16 (General Electric, Waukeshka, Wis) PET/CT scanner. First, PET/CT imaging was done in conjunction with a low-dose CT for attenuation correction. Patients were scanned from skull base to mid thigh and injected with an average of $11.0 \mathrm{mCi}{ }^{18} \mathrm{FDG}$. Immediately thereafter, a dedicated 
TABLE 1. Baseline patient characteristics

\begin{tabular}{|c|c|c|}
\hline \multirow[b]{2}{*}{ Characteristic } & \multicolumn{2}{|c|}{ Patients $(n=35)$} \\
\hline & $\mathbf{n}$ & $\%$ \\
\hline \multicolumn{3}{|l|}{ Gender } \\
\hline Male & 15 & 43 \\
\hline Female & 20 & 57 \\
\hline \multicolumn{3}{|l|}{ Age } \\
\hline$\leq 60 \mathrm{y}$ & 1 & 3 \\
\hline$>60-70 \mathrm{y}$ & 15 & 43 \\
\hline$>70-80 y$ & 14 & 40 \\
\hline$>80-90$ y & 4 & 11 \\
\hline$>90 \mathrm{y}$ & 1 & 3 \\
\hline \multicolumn{3}{|l|}{ PS (ECOG) } \\
\hline$\leq 2$ & 20 & 57 \\
\hline$\geq 3$ & 15 & 43 \\
\hline \multicolumn{3}{|l|}{ Reason for inoperability } \\
\hline $\begin{array}{l}\text { Poor pulmonary function } \\
\left(\mathrm{FEV}_{1} \text { and/or DLCO }<40 \%\right)\end{array}$ & 20 & 57 \\
\hline Other cardiopulmonary & 8 & 23 \\
\hline Other medical* & 3 & 9 \\
\hline Refusal of surgery & 4 & 11 \\
\hline \multicolumn{3}{|l|}{ Tumor size } \\
\hline$\leq 2 \mathrm{~cm}$ & 17 & 49 \\
\hline$>2-3 \mathrm{~cm}$ & 11 & 31 \\
\hline$>3-5 \mathrm{~cm}$ & 5 & 14 \\
\hline$>5$ & 2 & 6 \\
\hline \multicolumn{3}{|l|}{ AJCC T stage } \\
\hline $\mathrm{T} 1$ & 24 & 69 \\
\hline $\mathrm{T} 2$ & 10 & 28 \\
\hline $\mathrm{T} 3$ & 1 & 3 \\
\hline \multicolumn{3}{|l|}{ Tumor location } \\
\hline Central & 5 & 14 \\
\hline Peripheral & 30 & 86 \\
\hline \multicolumn{3}{|l|}{ Histology } \\
\hline Adenocarcinoma & 14 & 40 \\
\hline Squamous & 15 & 43 \\
\hline NSCLC (NOS) & 6 & 17 \\
\hline \multicolumn{3}{|l|}{ N0 status } \\
\hline $\mathrm{cNO}+$ & 22 & 63 \\
\hline $\mathrm{pNO} \ddagger$ & 13 & 37 \\
\hline EBUS & 5 & 14 \\
\hline Mediastinoscopy & 4 & 11 \\
\hline EBUS + mediastinoscopy & 4 & 11 \\
\hline
\end{tabular}

PS, Performance status; ECOG, Eastern Cooperative Oncology group; $F E V_{1}$, forced expiratory volume in 1 second; $D L C O$, diffusing capacity of carbon monoxide; $A J C C$, American Joint Commission on Cancer; NSCLC, non-small cell lung cancer; NOS, nitric oxide synthase; EBUS, endobronchial ultrasound. *One patient with AIDS and CD4 <200; one patient debilitated after cerebrovascular accident; one patient with Alzheimer disease. $\lceil\mathrm{cNO}=$ clinically staged hilum/mediastinum. $\ddagger \mathrm{pNO}=$ pathologically staged hilum/mediastinum.

contrast-enhanced diagnostic level chest CT was performed without moving the patient; this setup allowed image fusion of PET and contrastenhanced diagnostic chest CT. The contrast-enhanced diagnostic chest CT used $120 \mathrm{~mL}$ of Optiray 320 intravenous contrast (Tyco Health Care/ Mallinckrodt, Raleigh, NC). The contrast was timed to achieve optimal enhancement for liver and pulmonary vasculature.

To compare the utility of CT and PET/d-chest for detection of regional recurrence, and to eliminate potential bias introduced by having access to both PET and d-chest images/reports, we had each diagnostic chest CT scan read independently by a board-certified radiologist with specialization in chest imaging. PET images were uncoupled from the diagnostic CT images, and he/she was blinded to the PET result. CT response assessment was performed according to the modified version of the Response Evaluation Criteria in Solid Tumors (RECIST) Committee criteria used to assess tumor burden. It was by these parameters that response within the planning target volume was determined. Local failure was also characterized by recurrence within the treated lobe, consistent with definitions established by RTOG 0236 and ACOSOG Z4099 (American College of Surgeons Oncology Group). Regional failure was considered to have occurred if lymph nodes greater than $1 \mathrm{~cm}$ in short-axis diameter appeared in the expected lymphatic hilar or mediastinal drainage path of the primary tumor. All other recurrences were considered local or distant.

\section{Statistics}

Rates of regional recurrence-free survival (rRFS) were calculated for the entire patient group on the basis of the results of PET/d-chest and CT alone. Once a recurrence was suspected by a specific follow-up modality, subsequent studies of that same modality were not considered, irrespective of whether additional studies existed. In addition, the rate of biopsy-proven rRFS was determined by removing patients with clinically detected yet unbiopsied recurrences. Overall survival was calculated from first SBRT treatment until the time of last follow-up or death; the Social Security Death Index was searched in the event that a patient was lost to follow-up.

GraphPad Prism Statistical Software Package (GraphPad Software, La Jolla, Calif) was used to calculate rRFS, biopsy-proven rRFS, and overall survival using Mantle-Cox (log-rank) statistics; Kaplan-Meier curves were constructed for each.

\section{RESULTS}

The median follow-up time for the entire patient population was 12.8 months (range, 0.3-36.9 months). At the time of this report, 14 patients have died. The 1-year and 2-year rates of overall survival were $77 \%$ and $62 \%$, respectively (Figure 1).

Twenty $(57 \%)$ patients did not have any recurrence of their cancer. For the remainder of the patients, the first site of cancer recurrence was as follows: $10(28.6 \%)$ in regional lymph nodes (ie, hilar and/or mediastinal), 1 (2.8\%) in the central nervous system, $2(5.7 \%)$ with pleural dissemination, $1(2.8 \%)$ with an adrenal metastasis, and $1(2.8 \%)$ with recurrence in the same lobe as the primary tumor. One of the regional recurrences occurred simultaneously with a new nodule in the same treated lobe more than 24 months after initial treatment. No recurrences were detected within the planning target volume according to modified RECIST criteria. There was no difference in the rates of recurrence when stratified by $\mathrm{T}$ descriptor.

The rRFS was evaluated by 2 means: PET/d-chest and diagnostic CT alone. Regional recurrence was detected in 10 $(28.6 \%)$ patients when they were evaluated by PET/d-chest. These regional recurrences occurred between 2.9 and 29 months after treatment (median, 4.45 months). Diagnostic $\mathrm{CT}$ alone detected no regional recurrences after treatment. The 2-year rates of rRFS as evaluated by CT and PET/dchest are $100 \%$ and $52.1 \%$, respectively. Of note, 2 of the patients with regional recurrence detected by PET/d-chest did have pathologic staging before treatment; the remaining

\section{.}




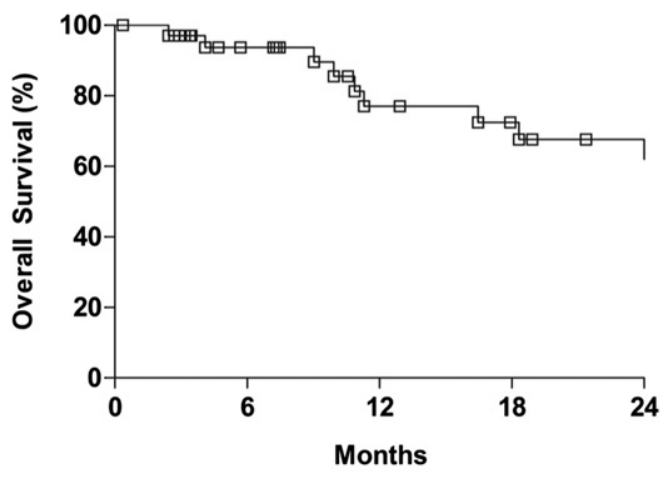

\begin{tabular}{l|c|c|c|c|c|}
\cline { 2 - 5 } No at risk: & 35 & 27 & 19 & 16 & 12 \\
\cline { 2 - 6 } OS (\%) & 100 & 94 & 77 & 72 & 62 \\
\hline
\end{tabular}

FIGURE 1. Kaplan-Meier curve for overall survival $(O S)$ for 35 patients treated with stereotactic body radiation therapy for medically inoperable early-stage non-small cell lung cancer. Median survival time for this group of patients is 28.6 months. Data summary table indicates number of patients at risk at $0,6,12,18$, and 24 months with corresponding percent overall survival for each time point.

8 were staged clinically as N0. Figure $2, A$, shows the comparison of Kaplan-Meier curves for rRFS based on followup imaging modality.

Six of the 10 patients with regional recurrences as determined by PET/d-chest did have biopsy confirmation; there was only 1 patient for whom an attempt at biopsy was unsuccessful; however, this patient did go on to receive salvage treatment (mediastinal chemoradiation) because of a high degree of suspicion based on imaging characteristics. The biopsy-proven regional recurrences occurred between 2.9 and 16.5 months after treatment (median, 4.95 months).
Only 1 patient with biopsy-proven regional recurrence was staged pathologically. The 1- and 2-year rates of biopsyproven rRFS as evaluated by PET/d-chest are $82.6 \%$ and $62 \%$, respectively. Figure $2, B$, shows the comparison of Kaplan-Meier curves for both biopsy-proven rRFS and clinical rRFS as determined by CT.

\section{DISCUSSION}

Stereotactic body radiotherapy for patients with medically inoperable, early stage, NO NSCLC has been established as a new standard of care. Before the wide acceptance of SBRT for this patient population, the only available options were conventionally fractionated radiation therapy, thermal ablation, or supportive care alone. Median survival time for patients with untreated stage I NSCLC ranges between 8 and 14 months with approximately $75 \%$ of patients dying of NSCLC. ${ }^{13}$ Conventionally fractionated radiotherapy provides only marginal benefit, with median survival times between 1.5 and 1.7 years. ${ }^{5}$ In contrast, SBRT yields a significant improvement in outcomes, with median survival times up to 48 months reported in multicenter trials. In our series of 35 patients treated with SBRT, the 2-year overall survival was $62 \%$ with a median survival time of 28.6 months. This is consistent with the results of the prospective multicenter RTOG 0236 trial, considered the modern benchmark for lung SBRT. ${ }^{7}$

In the early phase of our SBRT program, we noted a higher than expected incidence of recurrence within regional lymph nodes. On the basis of published reports, we expected $90 \%$ to $95 \%$ rRFS at 2 years, ${ }^{7}$ and in our patients rRFS was $52.1 \%$. We hypothesized that this was in part a result of enhanced detection owing to our routine use of PET/
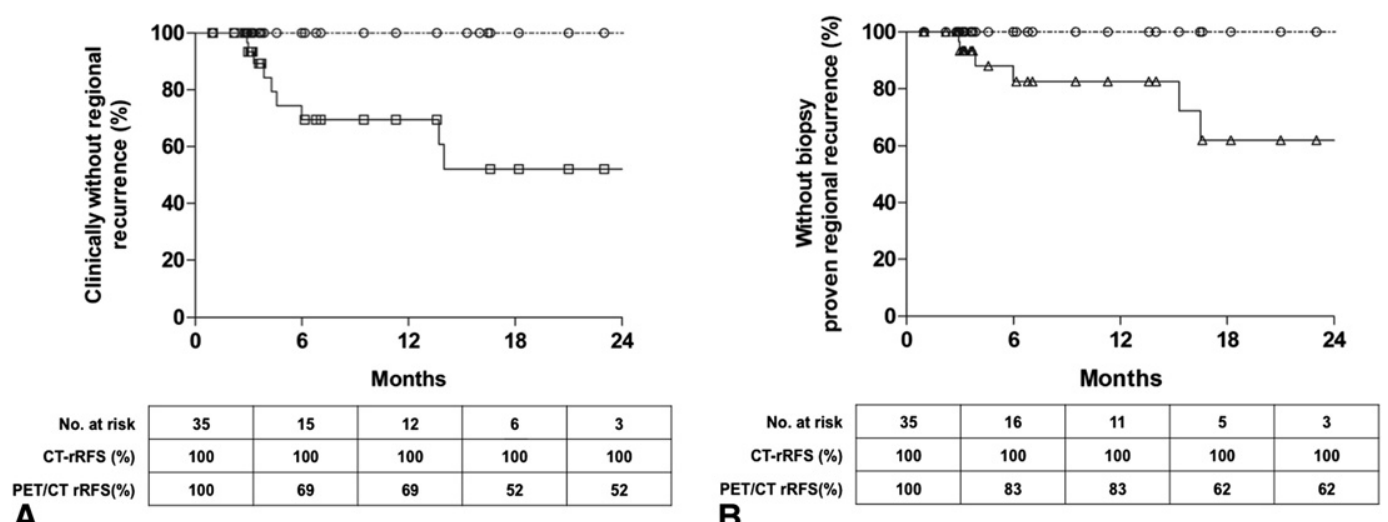

FIGURE 2. Kaplan-Meier curves comparing (A) rRFS for 35 patients treated with SBRT for early-stage NSCLC based on follow-up modality (A) and biopsy-proven rRFS for the same 35 patients (B). A, Open circles $(O)$ indicate rRFS as evaluated by CT and squares $(\square)$ by PET/d-chest. The difference between these 2 graphs is statistically significant $(P=.0045)$. B, Open circles $(O)$ indicate rRFS as evaluated by CT and triangles $(\triangle)$ indicate only regional recurrence that were picked up by PET/d-chest that were subsequently pathologically confirmed with biopsy. The difference between these 2 curves remains statistically significant $(P=.03)$. Data summary table in A and B indicate number of patients at risk at $0,6,12,18$, and 24 months with corresponding percent rRFS or biopsy-proven rRFS for each time point, respectively. $r R F S$, Regional recurrence-free survival; $S B R T$, stereotactic body radiation therapy; NSCLC, non-small cell lung cancer; $C T$, computed tomography; PET/d-chest, positron emission tomography combined with diagnostic chest computed tomography; PET, positron emission tomography. 

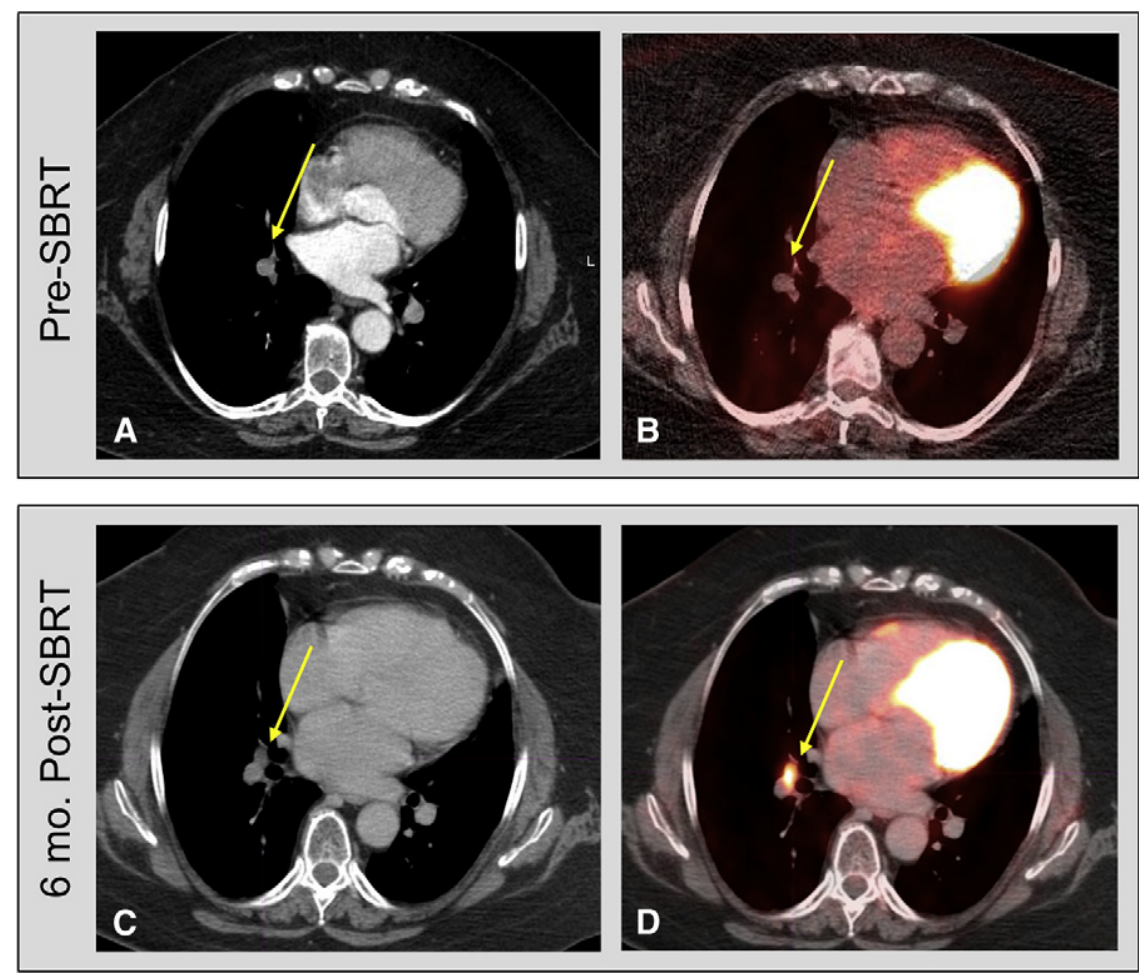

FIGURE 3. Representative CT and PET/d-chest images for patient 2 (see Table 2) with right hilar lymph node recurrence of NSCLC 6 months after SBRT treatment. A and B, Pretreatment CT and PET/CT images, respectively. C and D, Six-month posttreatment CT and PET/CT images from the same PET/dchest examination. The yellow arrow notes the same anatomic location in the right hilum on all 4 representative images. Before treatment there was no enlarged or hypermetabolic lymph node in this region. On the post-treatment CT scan $(\mathrm{C})$, the lymph node noted by the yellow arrow measures $8 \mathrm{~mm}$ in short-axis diameter. On the basis of CT evaluation criteria, this was believed to be benign/inflammatory related to prior SBRT treatment. On the posttreatment PET/d-chest (D), the 8-mm lymph node believed to be benign on CT was intensely hypermetabolic with SUVmax $=5.2$. Subsequent EBUS-guided FNA biopsy confirmed recurrent NSCLC, and this patient was treated with salvage chemo/RT (66 Gy with carboplatin/paclitaxel) and is disease free 24 months after salvage therapy. CT, Computed tomography; PET, positron emission tomography; PET/d-chest, positron emission tomography combined with diagnostic chest computed tomography; NSCLC, non-small cell lung cancer; SBRT, stereotactic body radiation therapy; SUVmax, maximum standardized uptake value; $E B U S$, endobronchial ultrasound; $F N A$, fine needle aspiration; $R T$, radiotherapy.

d-chest, rather than $\mathrm{CT}$ alone. Figure 3 demonstrates an example of a nodal recurrence detected by PET/d-chest that would have been missed by anatomic CT imaging alone. Because of the manner in which our follow-up scans were performed (as combined diagnostic chest CT with contrast, and PET) we were able to separate the studies and reinterpret the CT component without knowledge or bias from the PET and report rRFS separately as determined by each modality. To our knowledge, this type of analysis with internal controls has not been performed previously. It allowed us to truly distill the added value of biologic imaging after SBRT. Most prior studies have focused on the utility of PET for detection of local recurrence within the target volume only. ${ }^{8,9,14}$ Inasmuch as a significant proportion of patients demonstrate elevated FDG uptake within the target volume for several months after SBRT, the value of PET, especially within the first 6 months after treatment, is controversial. Data presented here, however, clearly indicate an important role for PET as the optimal mode of surveillance for potentially salvageable regional recurrence. Evaluation of the regional lymphatics is particularly relevant when one considers that many patients receiving SBRT undergo radiographic staging only, whereas surgical resection offers the opportunity of pathologic staging at the time of resection.

There were no detected regional recurrences when follow-up evaluation was limited to CT alone. The addition of PET aided in the detection of 10 regional recurrences in the same 35 patients. Table 2 outlines details of these recurrences. Because of the known and clinically relevant falsepositive rate of PET/CT for mediastinal staging, we sought pathologic confirmation of suspected recurrences whenever possible. $^{15}$ The majority $(60 \%)$ of the recurrences were confirmed pathologically. Even when one considers only those recurrences that were pathologically confirmed, sensitivity for regional recurrence remains significantly improved with the addition of PET to diagnostic CT imaging. It is unclear whether earlier detection of regional recurrences will significantly affect overall survival in this medically frail patient population, where few are fit for salvage 
TABLE 2. Characteristics of patients with regional recurrence

\begin{tabular}{|c|c|c|c|c|c|c|c|c|c|c|c|c|c|}
\hline \multicolumn{4}{|c|}{ Demographic } & \multicolumn{4}{|c|}{ Tumor/staging } & \multirow{2}{*}{$\begin{array}{c}\text { Treatment } \\
\\
\text { SBRT } \\
\left(\text { Gy }\left[\mathrm{BEDGy}_{10}\right]\right)\end{array}$} & \multicolumn{3}{|c|}{ Recurrence } & \multicolumn{2}{|c|}{ Status } \\
\hline$\underline{P t}$ & Age & Sex & $\begin{array}{c}\text { ECOG } \\
\text { PS } \\
\end{array}$ & $\begin{array}{c}\text { AJCC } \\
\text { T stage }\end{array}$ & $\begin{array}{l}\text { LD } \\
(\mathrm{cm})\end{array}$ & $\begin{array}{c}\text { Tumor } \\
\text { location }\end{array}$ & $\begin{array}{c}\text { Invasive } \\
\text { mediastinal } \\
\text { staging? }\end{array}$ & & $\begin{array}{c}\text { rRFS } \\
(\mathrm{mo}) \\
\end{array}$ & $\begin{array}{c}\text { Biopsy- } \\
\text { proven }(\mathbf{Y} / \mathbf{N})\end{array}$ & $\begin{array}{c}\text { Salvage } \\
\text { treatment }\end{array}$ & $\begin{array}{c}\text { Alive/ } \\
\text { dead }\end{array}$ & $\begin{array}{c}\text { Survival } \\
(\mathrm{mo}) \\
\end{array}$ \\
\hline 1 & 61 & $\mathrm{~F}$ & 2 & $1 b$ & 2.9 & Peripheral & None & 56 [134.4] & 16.5 & $\mathrm{Y}$ & None & Dead & 31.7 \\
\hline 2 & 64 & $\mathrm{~F}$ & 1 & 1a & 1.4 & Peripheral & None & 56 [134.4] & 6 & Y & CRT & Alive & 36.9 \\
\hline 3 & 73 & $\mathrm{~F}$ & 1 & $2 b$ & 5.3 & Peripheral & None & 56 [134.4] & 14 & $\mathrm{~N}^{*}$ & CRT & Dead & 34 \\
\hline 4 & 53 & $\mathrm{~F}$ & 2 & $1 \mathrm{a}$ & 1.6 & Peripheral & MED/EBUS & 56 [134.4] & 29.2 & $\mathrm{~N}$ & None & Alive & 32.8 \\
\hline 5 & 68 & M & 1 & $1 \mathrm{a}$ & 1.2 & Peripheral & None & 56 [134.4] & 3 & $\mathrm{Y}$ & Chemo & Dead & 24 \\
\hline 6 & 69 & $\mathrm{~F}$ & 1 & $1 \mathrm{a}$ & 1.5 & Peripheral & None & 60 [180] & 15.3 & $\mathrm{Y}$ & CRT & Dead & 28.6 \\
\hline 7 & 81 & $\mathrm{~F}$ & 2 & $1 b$ & 2.4 & Peripheral & None & 50 [112.5] & 4.6 & $\mathrm{~N}$ & None & Dead & 18.3 \\
\hline 8 & 63 & $\mathrm{~F}$ & 2 & $1 b$ & 3.3 & Peripheral & EBUS & 56 [134.4] & 2.9 & $\mathrm{Y}$ & None & Dead & 9.9 \\
\hline 9 & 71 & $\mathrm{~F}$ & 3 & $1 \mathrm{a}$ & 2 & Peripheral & None & 60 [180] & 3.9 & Y & CRT & Dead & 26.1 \\
\hline 10 & 77 & $\mathrm{~F}$ & 3 & $2 b$ & 5.4 & Peripheral & None & $50[100]$ & 3.3 & $\mathrm{~N}$ & None & Dead & 11.3 \\
\hline
\end{tabular}

$E C O G$, Eastern Cooperative Oncology group; $P S$, performance status; AJCC, American Joint Commission on Cancer; $L D$, longest diameter of the tumor on the pretreatment chest computed tomogram; $S B R T$; stereotactic body radiation therapy; $B E D G y_{10}$, biologically equivalent radiation therapy dose determined using the linear quadratic equation with $\alpha /$ $\beta=10 ; r R F S$, regional recurrence free survival; $C R T$, chemoradiotherapy; $M E D$, mediastinoscopy; EBUS, endobronchial ultrasound. *This patient received salvage treatment even though pathologic confirmation of recurrence was not obtained owing to very high clinical suspicion.

treatment. However, as indications for SBRT are expanded to perhaps include the high-risk yet operable patients for whom salvage treatment may be feasible, early detection of locoregional recurrence becomes highly relevant. Of note, all of the regional cancer recurrences in our patients occurred in the absence of distant metastatic cancer, further increasing the chance for successful salvage. Five of these patients received no salvage treatment because they were candidates for neither extended-field thoracic reirradiation nor systemic chemotherapy.

Various strategies have been used for the treatment of locoregional recurrence after definitive treatment of early-stage NSCLC. Conventionally fractionated radiation therapy after initial resection has been shown to result in some long-term survivors, justifying a second attempt at definitive local therapy. ${ }^{16,17}$ Conversely, salvage surgical resection after conventionally fractionated definitive radiation therapy is warranted in selected cases. ${ }^{18}$ Also, salvage surgical resection, including anatomic lobectomy and segmentectomy, can be safely performed after SBRT as well. ${ }^{19}$ This is confirmed by experience at our own institution. Reirradiation is also feasible in patients with longer disease-free intervals and a reasonable performance status. ${ }^{20}$ Newer evidence suggests that definitive conventionally fractionated radiation therapy (or chemoradiotherapy) can be performed for hilar and/or mediastinal regional recurrence after SBRT with some success. ${ }^{21}$ Four of our patients who had regional lymph node recurrences without local recurrence subsequently underwent salvage treatment consisting of mediastinal/hilar radiation therapy combined with chemotherapy in a similar fashion to previously reported salvage treatments. ${ }^{21}$ These experiences justify more sensitive surveillance of patients undergoing SBRT, especially those with favorable performance status, with periodic PET/d-chest as salvage may be possible. A nihilistic approach to locoregional recurrence is inadequate.

Regional recurrence is relatively uncommon in prior studies of lung SBRT. In the RTOG 0236 study, only 2 patients of 55 had regional recurrence, at 33 and 36 months. PET was not mandated. It is unclear whether these recurrences might have been detected earlier had PET scanning been performed or whether additional regional recurrences had gone undetected before either progression to disseminated disease or termination of follow-up. Other variables may have contributed to the higher than expected regional recurrence rates in the current study. Inaccurate clinical staging may have been a factor, especially in those patients with early ( $<6$ months after SBRT) recurrences. Initial radiographic staging was performed with CT, PET, and brain $\mathrm{CT}$ or magnetic resonance imaging. Many of the pretreatment scans were performed at outside referring institutions and could not be repeated owing to insurance issues. As such, the scan timing, technique, and accuracy of the readings were not standardized. Invasive staging with mediastinoscopy, EBUS, and/or endoscopic esophageal ultrasound were aggressively yet selectively performed as a function of tumor size, mediastinal/hilar nodal size, patient performance status, and patient preference. The importance of invasive staging before nonoperative treatments is underemphasized, because we know that EBUS discovers mediastinal disease in roughly $10 \%$ of PET/CT-negative and $16 \%$ of CT-negative patients. ${ }^{22}$ This is especially important in patients with central tumors, where $50 \%$ harbor nodal disease regardless of T stage. ${ }^{23}$ Another possible factor could have been unintentional underdosage of the planning target volume because of inherent inaccuracies in the Ray-Tracing dose-calculation algorithm used for the first 18 months of our program. In 2010 the extreme variability in actual dose delivered (vs intended dose) with this technique was 
reported and cautioned against its routine use when treating lung tumors. Subsequently, we used the more robust Monte Carlo algorithm. ${ }^{24}$ Longer follow-up will be necessary to determine whether this was truly a factor in this series.

In summary, our series demonstrates that PET/d-chest enhances the detection of regional progression of NSCLC after SBRT over currently recommended radiographic strategies. The superiority of the addition of PET to diagnostic $\mathrm{CT}$ is critically important inasmuch as it pertains to ongoing and planned prospective studies evaluating SBRT for the treatment of patients with operable NSCLC. Since patients enrolled in these studies are likely to be healthier and can potentially tolerate salvage treatment with surgery or chemoradiotherapy, early detection is paramount. Delays may result in further cancer dissemination with no chance for salvage and ultimately compromise survivals. Although our data cannot be used to determine the optimal frequency of posttreatment follow-up with PET, it is essential that it be combined with anatomic imaging to enhance the early detection of regional cancer recurrences. In conclusion, we strongly recommend that all prospective and/or cooperative group studies of SBRT for the treatment of patients with borderline-operable or operable NSCLC be amended to require PET surveillance along with diagnostic $\mathrm{CT}$ and that $\mathrm{PET} / \mathrm{d}$-chest follow-up should be considered in routine lung SBRT practice.

\section{References}

1. Inoue T, Shimizu S, Onimaru R, Takeda A, Onishi H, Nagata Y, et al. Clinical outcomes of stereotactic body radiotherapy for small lung lesions clinically diagnosed as primary lung cancer on radiologic examination. Int J Radiat Oncol Biol Phys. 2009;75:683-7.

2. Fakiris AJ, McGarry RC, Yiannoutsos CT, Papiez L, Williams M, Henderson MA, et al. Stereotactic body radiation therapy for early-stage nonsmall-cell lung carcinoma: four-year results of a prospective phase II study. Int J Radiat Oncol Biol Phys. 2009;75:677-82.

3. McGarry RC, Papiez L, Williams M, Whitford T, Timmerman RD. Stereotactic body radiation therapy of early-stage non-small-cell lung carcinoma: phase I study. Int J Radiat Oncol Biol Phys. 2005;63:1010-5.

4. Timmerman R, McGarry R, Yiannoutsos C, Papiez L, Tudor K, DeLuca J, et al. Excessive toxicity when treating central tumors in a phase II study of stereotactic body radiation therapy for medically inoperable early-stage lung cancer. J Clin Oncol. 2006;24:4833-9.

5. Fang LC, Komaki R, Allen P, Guerrero T, Mohan R, Cox JD. Comparison of outcomes for patients with medically inoperable Stage I non-small-cell lung cancer treated with two-dimensional vs. three-dimensional radiotherapy. Int J Radiat Oncol Biol Phys. 2006;66:108-16.

6. Potters L, Kavanagh B, Galvin JM, Hevezi JM, Janjan NA, Larson DA, et al. American Society for Therapeutic Radiology and Oncology; American College of Radiology. American Society for Therapeutic Radiology and Oncology (ASTRO) and American College of Radiology (ACR) practice guideline for the performance of stereotactic body radiation therapy. Int J Radiat Oncol Biol Phys. 2010;76:326-32.
7. Timmerman R, Paulus R, Galvin J, Michalski J, Straube W, Bradley J, et al. Stereotactic body radiation therapy for inoperable early stage lung cancer. JAMA. 2010;303:1070-6.

8. Hoopes DJ, Tann M, Fletcher JW, Forquer JA, Lin PF, Lo SS, et al. FDG-PET and stereotactic body radiotherapy (SBRT) for stage I non-small-cell lung cancer. Lung Cancer. 2007;56:229-34.

9. Henderson MA, Hoopes DJ, Fletcher JW, Lin PF, Tann M, Yiannoutsos CT, et al. A pilot trial of serial $18 \mathrm{~F}$-fluorodeoxyglucose positron emission tomography in patients with medically inoperable stage I non-small-cell lung cancer treated with hypofractionated stereotactic body radiotherapy. Int J Radiat Oncol Biol Phys. 2010;76:789-95.

10. van Loon J, Grutters J, Wanders R, Boersma L, Oellers M, Dingemans AM, et al Follow-up with 18FDG-PET-CT after radical radiotherapy with or without chemotherapy allows the detection of potentially curable progressive disease in nonsmall cell lung cancer patients: a prospective study. Eur J Cancer. 2009;45: 588-95.

11. Verschakelen JA, De Wever W, Bogaert J. Role of computed tomography in lung cancer staging. Curr Opin Pulm Med. 2004;10:248-55.

12. Schrevens L, Lorent N, Dooms C, Vansteenkiste J. The role of PET scan in diagnosis, staging, and management of non-small cell lung cancer. Oncologist. 2004; 9:633-43.

13. Raz DJ, Zell JA, Ou SH, Gandara DR, Anton-Culver H, Jablons DM. Natural history of stage I non-small cell lung cancer: implications for early detection. Chest. 2007;132:193-9.

14. Zhang X, Liu H, Balter P, Allen PK, Komaki R, Pan T, et al. Positron emission tomography for assessing local failure after stereotactic body radiotherapy for non-small-cell lung cancer. Int J Radiat Oncol Biol Phys. 2012;83: 1558-65.

15. Darling GE, Maziak DE, Inculet RI, Gulenchyn KY, Driedger AA, Ung YC, et al Positron emission tomography-computed tomography compared with invasive mediastinal staging in non-small cell lung cancer: results of mediastinal staging in the early lung positron emission tomography trial. $J$ Thorac Oncol. 2011;6: 1367-72.

16. Kelsey CR, Clough RW, Marks LB. Local recurrence following initial resection of NSCLC: salvage is possible with radiation therapy. Cancer J. 2006;12:283-8.

17. Cai XW, Xu LY, Wang L, Hayman JA, Chang AC, Pickens A, et al. Comparative survival in patients with postresection recurrent versus newly diagnosed nonsmall-cell lung cancer treated with radiotherapy. Int J Radiat Oncol Biol Phys. 2010;76:1100-5.

18. Bauman JE, Mulligan MS, Martins RG, Kurland BF, Eaton KD, Wood DE. Salvage lung resection after definitive radiation ( $>59$ Gy) for non-small cell lung cancer: surgical and oncologic outcomes. Ann Thorac Surg. 2008;86:1632-8; discussion 1638-9.

19. Chen F, Matsuo Y, Yoshizawa A, Sato T, Sakai H, Bando T, et al. Salvage lung resection for non-small cell lung cancer after stereotactic body radiotherapy in initially operable patients. J Thorac Oncol. 2010;5:1999-2002.

20. Tada T, Fukuda H, Matsui K, Hirashima T, Hosono M, Takada Y, et al. Nonsmall-cell lung cancer: reirradiation for loco-regional relapse previously treated with radiation therapy. Int J Clin Oncol. 2005;10:247-50.

21. Manabe Y, Shibamoto Y, Baba F, Murata H, Ogino S, Ayakawa S, et al. Definitive radiotherapy for hilar or mediastinal lymph node metastases after stereotactic body radiotherapy for stage I non-small cell lung cancer. Int J Radiat Oncol Biol Phys. 2010;78(3S):S181.

22. Sarwate D, Sarkar S, Krimsky WS, Burgan CM, Patel K, Evans R, et al. Optimization of mediastinal staging in potential candidates for stereotactic radiosurgery of the chest. J Thorac Cardiovasc Surg. 2012;144:81-6.

23. Ketchedjian A, Daly BD, Fernando HC, Florin L, Hunter CJ, Morelli DM, et al Location as an important predictor of lymph node involvement for pulmonary adenocarcinoma. J Thorac Cardiovasc Surg. 2006;132:544-8.

24. Wilcox EE, Daskalov GM, Lincoln H, Shumway RC, Kaplan BM, Colasanto JM. Comparison of planned dose distributions calculated by Monte Carlo and RayTrace algorithms for the treatment of lung tumors with cyberknife: a preliminary study in 33 patients. Int J Radiat Oncol Biol Phys. 2010;77:277-84. 